\section{Case Reports in Dermatology}

Case Rep Dermatol 2017;9:40-43

DOI: $10.1159 / 000477375$

Published online: June 14, 2017

(C) 2017 The Author(s)

Published by S. Karger AG, Basel

www.karger.com/cde

This article is licensed under the Creative Commons Attribution-NonCommercial 4.0 International License (CC BY-NC) (http://www.karger.com/Services/OpenAccessLicense).

Usage and distribution for commercial purposes requires written permission.

\title{
Erythema Gyratum Repens-Like Purpura in a Patient with Sjögren Syndrome
}

\author{
Miyuki Fukunaga $^{a}$ Kazutoshi Harada $^{a}$ Kenichiro Mae ${ }^{a}$ \\ Kanae Wakamatsu ${ }^{a} \quad$ Noriko Kiriyama $^{\mathrm{a}}$ b Ryoji Tsuboi $^{\mathrm{a}} \quad$ Yukari Okubo $^{\mathrm{a}}$ \\ ${ }^{a}$ Department of Dermatology, Tokyo Medical University, Tokyo, Japan; ${ }^{b}$ Department of \\ Dermatology, Akishima Hospital, Tokyo, Japan
}

\section{Keywords}

Annular erythema - Leukocytoclastic vasculitis · Purpura · Hypergammaglobulinemia · Sjögren syndrome

\section{Abstract}

The etiology of purpura in Sjögren syndrome (SS) includes cryoglobulinemia, hypergammaglobulinemia, and leukocytoclastic vasculitis (LCV). The clinical symptoms of LCV associated with SS comprise palpable or nonpalpable purpura and urticarial vasculitis. Here, we report a case of LCV presenting as erythema gyratum repens (EGR)-like purpura in a 62-year-old woman with rheumatoid arthritis and SS. EGR-like skin lesions, characterized by annular lesions with expanding concentric pattern and coalescing to form a zebra-like pattern or grain of wood pattern, can appear in various autoimmune conditions; however, EGR-like eruption in SS is extremely rare. On the basis of the expansion pattern, we considered that the EGRlike purpura in this case was elicited by urticarial vasculitis accompanied by SS. 


\section{Introduction}

Erythema gyratum repens (EGR)-like skin lesions, characterized by centrifugal eruption, can appear in various autoimmune conditions. Herein, we report a case of leukocytoclastic vasculitis (LCV) presenting as EGR-like purpura in a patient with Sjögren syndrome (SS). EGR-like purpura in this case may have been elicited by urticarial vasculitis accompanied by SS.

\section{Case Report}

A 62-year-old woman consulted our department for a curious purpura demonstrating centrifugal expansion. Although she had been diagnosed as having rheumatoid arthritis and prescribed methotrexate $(8 \mathrm{mg} /$ week), this treatment was discontinued because of side effects, leading to stomatitis and inappetence. One month before her visit to another clinic, bizarre purpuric eruptions appeared. Treatment with oral prednisolone $(40 \mathrm{mg} / \mathrm{day})$ was started for the lesions. She was successfully treated with the oral steroid; however, purpura recurred after reducing the drug. When she was first seen in our hospital, the treatment with oral prednisolone $(20 \mathrm{mg} /$ day) was restarted. Physical examination revealed concentric purpuric patches and plaques on the lower limbs and the buttock (Fig. 1a). The purpura expanded centrifugally to form patch or plaques and some of them coalesced to form a zebra like pattern (Fig. 1b). The color of the concentric annular purpura sequentially turned from reddish purple to dark purple and then to yellowish brown (Fig. 1b). Erythema, papules, or wheals had never been observed in the course of the eruptions. A biopsy specimen taken from a purpuric patch revealed perivascular infiltration of a few neutrophils, nuclear dust, extravasated red blood cells, and swelling of the endothelial cells in the papillary dermis (Fig. 1c), suggesting the presence of LCV. Immunofluorescence studies showed no deposition of immunoglobulins or complements in the vessel walls. Laboratory examination revealed positive antinuclear antibody (specked pattern with titer 1/80), positive anti-SS-A and antiCCP antibody, increased erythrocyte sedimentation rate $(109 \mathrm{~mm} / \mathrm{h})$, and high IgG titer $(2,080 \mathrm{mg} / \mathrm{dL}$; normal, $870-1,700 \mathrm{mg} / \mathrm{dL})$. Serum C3 and C4 concentrations were normal and no cryoglobulin was detected. Immune complexes (C1q) and antineutrophil cytoplasmic antibodies(c-ANCA, p-ANCA) were within the normal ranges. No bleeding lesions were observed by endoscopy, and no malignant tumor was observed by CT scan. The Schirmer test and the fluorescent test in both eyes as well as the chewing gum test were positive.

A lip biopsy specimen showed prominent infiltration of lymphocytes around the labial salivary ducts. Based on these finding, it was decided that the bizarre purpuric eruption in this patient was caused by LCV complicated by rheumatoid arthritis and SS. The dose of systemic steroids was increased to $20 \mathrm{mg} /$ day and the skin lesions completely resolved within one month and a half, leaving brown pigmentation. The dose was tapered thereafter without relapse. The skin lesions have not relapsed for 17 month with prednisolone $5 \mathrm{mg} /$ day.

\section{Discussion}

Purpura is one of the major dermatologic manifestations of SS [1]. The etiology of purpura in SS includes cryoglobulinemia, hypergammaglobulinemia, and LCV. The laboratory test for the present case detected no cryoglobulin, suggesting that the purpura in this patient 
was not caused by cryoglobulin. The patient had hypergammaglobulinemia; however, the titer of serum gamma globulin was slightly elevated. The hypergammaglobulinemic purpura was induced by serum hyperviscosity due to a high IgG titer. The characteristic clinical feature of hypergammaglobulinemic purpura is brownish pigmentation [2]. The laboratory findings and clinical manifestation indicated that the etiology of purpura in this case was not hypergammaglobulinemia. The clinical symptoms of LCV associated with SS comprise palpable or nonpalpable purpura and urticarial vasculitis [1]. Although our patient showed spot-sized purpura, the eruption had an expanded concentric pattern and coalesced to form a zebra-like pattern or grain of wood pattern (Fig. 1a). Urticarial vasculitis is a clinicopathological entity characterized by urticarial erythema with LCV. There are rare case reports of urticarial vasculitis presenting clinically as EGR-like eruption [3,4]. Additionally, a case of EGR-like erythema in a patient with SS has been reported [5]. On the basis of the expansion pattern and the distribution of the skin eruptions, we considered that the EGR-like purpura in this case was elicited by urticarial vasculitis accompanied by SS. The skin lesions of urticarial vasculitis include wheals, erythema, purpura, and pigmentation, whereas the eruptions in this case exclusively consisted of purpura. The patient received systemic steroid for the treatment of her rheumatoid arthritis. Application of systemic steroid might contribute to the abrogation of erythemas or wheals.

The conditions to be considered in the differential diagnosis of annular purpura include the following: erythema annulare centrifugum, erythema marginatum rheumaticum, and Majocchi disease. Erythema annulare centrifugum is an erythematous disorder characterized by annular erythema without scale. However, purpura caused by leukocytoclastic angiitis is never associated with this skin disorder. Erythema marginatum rheumaticum manifesting as erythematous annular papules is followed by rheumatic fever due to group A streptococcal infection. These erythematous disorders were distinguished by histologic examination and past history in this case. Majocchi disease is a form of pigmented purpuric dermatosis characterized by papular telangiectasia and annular purpura. The dermatologic examination revealed annular purpura in the lower extremities of this patient, thus Majocchi disease should be differentiated from the purpuric lesions in the present case. The purpura in this case showed a concentric pattern, whereas purpura in Majocchi disease never expands centrifugally. Moreover, papular or macular telangiectasia were not observed in this case. These clinical features allowed us to exclude the diagnosis of Majocchi disease.

\section{Statement of Ethics}

The authors have no ethical conflict to declare.

\section{Disclosure Statement}

The authors have no conflict of interest to declare. 


\section{Case Reports in Dermatology}

\section{References}

1 Ramos-Casals M, Anaya JM, García-Carrasco M, Rosas J, Bové A, Claver G, Diaz LA, Herrero C, Font J: Cutaneous vasculitis in primary Sjögren syndrome: classification and clinical significance of 52 patients. Medicine (Baltimore) 2004;83:96-106.

-2 Katayama I: Clinical analysis of recurrent hypergammaglobulinemic purpura associated with Sjögren syndrome. J Dermatol 1995;22:186-190.

-3 Dermitsu T, Sasaki K, Iida E, Azuma R, Umemoto N, Kakurai M, Yoneda K: Urticarial vasculitis presenting as erythema gyratum repens-like eruption. J Eur Acad Dermatol Venereol 2009;23:215-217.

4 Spierings NM, Natkunarajah J: Leucocytoclastic vasculitis presenting as an erythema gyratum repenslike eruption. Clin Exp Dermatol 2016;41:320-322.

-5 Matsumura T, Kumakiri M, Sato-Matsumura KC, Ohkawara A: Erythema gyratum repens-like eruption in a patient with Sjögren syndrome. Acta Derm Venereol 1995;75:327.
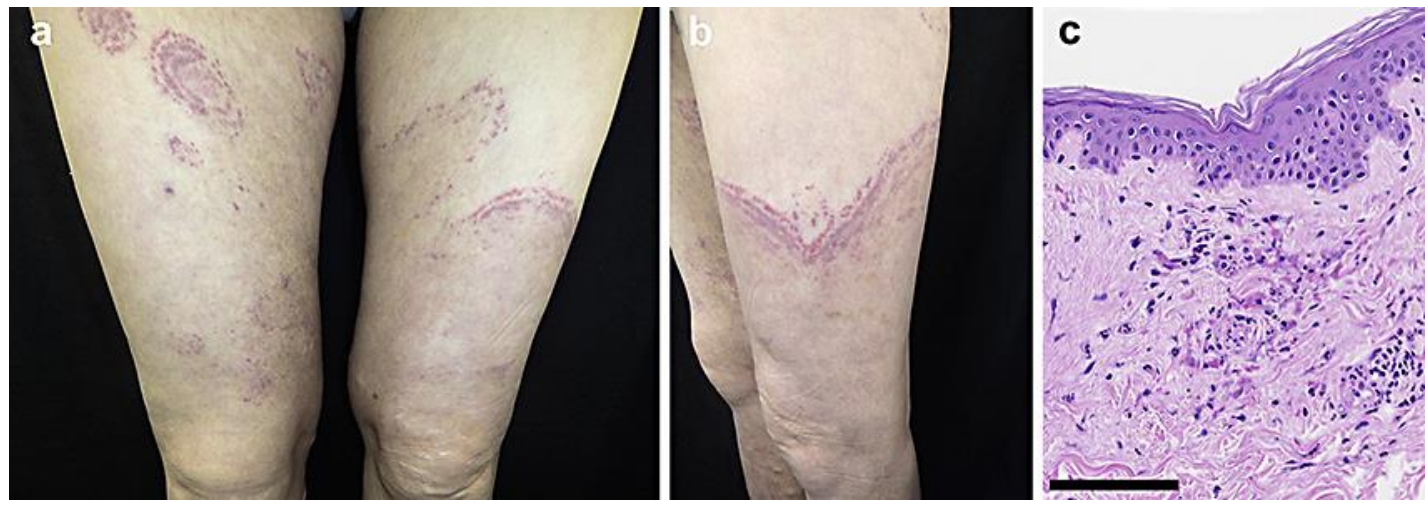

Fig. 1. a Concentric annular purpura in the lower extremities. b In left thigh, the lesions were formed as circinate purpura and presented scarlet, dark purple, and light yellow from the proximal edges. c Extravasation of erythrocytes and nuclear dust associated with endothelial cell swelling were observed at histologic examination. Hematoxylin-eosin. Scale bar, $100 \mu \mathrm{m}$. 